\title{
HIGH-PRECISION RADIO AND INFRARED ASTROMETRY OF LSPM J1314+1320AB. I. PARALLAX, PROPER MOTIONS, AND LIMITS ON PLANETS
}

\author{
Jan Forbrich $^{1,2}$, Trent J. Dupuy ${ }^{3}$, Mark J. Reid $^{2}$, Edo Berger ${ }^{2}$, Aaron Rizzuto $^{3}$, Andrew W. Mann ${ }^{3}$, Michael C. Liu ${ }^{4}$, \\ Kimberly Aller ${ }^{4}$, AND Adam L. Kraus ${ }^{3}$ \\ ${ }^{1}$ University of Vienna, Department of Astrophysics, Türkenschanzstr. 17, A-1180 Vienna, Austria \\ ${ }^{2}$ Harvard-Smithsonian Center for Astrophysics, 60 Garden Street, Cambridge, MA 02138, USA \\ ${ }^{3}$ The University of Texas at Austin, Department of Astronomy, 2515 Speedway C1400, Austin, TX 78712, USA \\ ${ }^{4}$ Institute for Astronomy, University of Hawai'i, 2680 Woodlawn Drive, Honolulu, HI 96822, USA \\ Received 2016 April 4; revised 2016 April 28; accepted 2016 April 28; published 2016 August 3
}

\begin{abstract}
We present multi-epoch astrometric radio observations with the Very Long Baseline Array (VLBA) of the young ultracool-dwarf binary LSPM J1314+1320AB. The radio emission comes from the secondary star. Combining the VLBA data with Keck near-infrared adaptive-optics observations of both components, a full astrometric fit of parallax ( $\pi_{\mathrm{abs}}=57.975 \pm 0.045 \mathrm{mas}$, corresponding to a distance of $\left.d=17.249 \pm 0.013 \mathrm{pc}\right)$, proper motion $\left(\mu_{\alpha \cos }\right.$

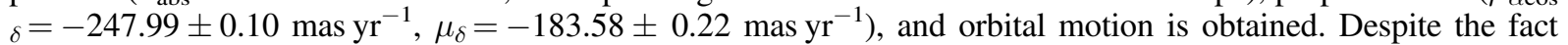
that the two components have nearly identical masses to within $\pm 2 \%$, the secondary's radio emission exceeds that of the primary by a factor of $\gtrsim 30$, suggesting a difference in stellar rotation history, which could result in different magnetic field configurations. Alternatively, the emission could be anisotropic and beamed toward us for the secondary but not for the primary. Using only reflex motion, we exclude planets of mass $0.7-10 M_{\text {jup }}$ with orbital periods of 600-10 days, respectively. Additionally, we use the full orbital solution of the binary to derive an upper limit for the semimajor axis of 0.23 au for stable planetary orbits within this system. These limits cover a parameter space that is inaccessible with, and complementary to, near-infrared radial velocity surveys of ultracool dwarfs. Our absolute astrometry will constitute an important test for the astrometric calibration of Gaia.
\end{abstract}

Key words: binaries: general - radio continuum: stars - stars: individual (LSPM J1314+1320) - stars: low-mass techniques: high angular resolution

\section{INTRODUCTION}

Since the discovery of non-thermal radio emission from ultracool dwarfs (Berger et al. 2001), where following Kirkpatrick et al. (1997), the term ultracool dwarfs here refers to spectral types M7 or later, and the detection of a nearby ultracool dwarf using Very Long Baseline Interferometry (VLBI; Forbrich \& Berger 2009), the prospect of using absolute VLBI astrometry to measure parallax and proper motion, and to search for residual reflex motions due to otherwise unseen planets, has been intriguing. Additionally, precise astrometry that can reveal the orbital evolution of nearby young multiple systems is of particular interest, since complete orbit solutions are a reliable method to determine masses, which can be used to test theoretical models of substellar systems (Baraffe et al. 2015; Dupuy et al. 2015). Only a few young binaries with very-low-mass components currently have reliable mass estimates.

While planets around $\mathrm{M}$ dwarfs are thought to be ubiquitous (e.g., Dressing \& Charbonneau 2015), the search for them is increasingly difficult for ultracool dwarfs. Planet searches toward stars of very low mass are usually carried out using transit or radial velocity (RV) techniques. For ultracool dwarfs, RV precisions of only $100-300 \mathrm{~m} \mathrm{~s}^{-1}$ can be reached when using high-resolution near-infrared spectra of slowly rotating stars (Blake et al. 2010), and the precision is lower for rapidly rotating stars. Given the difficulties of RV monitoring, astrometric observations provide an attractive alternative.

With our first VLBI detection of an ultracool dwarf (Forbrich \& Berger 2009), we pointed out the feasibility of looking for orbiting extrasolar planets by detecting their reflex motion with VLBI astrometry. The first astrometric monitoring campaign toward TVLM 513-46546 confirmed the feasibility with significant upper limits on the presence of extrasolar planets (Forbrich et al. 2013), excluding (at a significance of $3 \sigma$ ) planets of masses and orbital periods ranging from $3.8 M_{\text {jup }}$ with an orbital period of 16 days to $0.3 M_{\text {jup }}$ with an orbital period of 710 days. Previously, such searches had been carried out for early M-type dwarfs (e.g., Bower et al. 2009). The principal advantage of VLBI observations would be to obtain absolute parallax and proper motions (tied to an extragalactic reference frame). Astrometric observations can also be carried out in the optical wavelength range with $10 \mathrm{~m}$ class telescopes, but require conversion of relative to absolute parallax (e.g., Sahlmann et al. 2016).

Schlieder et al. (2014) presented LSPM J1314+1320AB as a potential "benchmark" binary for the determination of substellar masses to calibrate evolutionary models at young ages. Designations of primary and secondary components are based on infrared magnitude. This system has a high proper motion (e.g., Lépine \& Shara 2005). Law et al. (2006) estimated the spectral type as a late $\mathrm{M}$ dwarf from multi-band photometry and detected a companion at a separation of 0 "' 13 from optical imaging. An optical astrometric parallax measurement was obtained by Lépine et al. (2009) who found $\pi_{\mathrm{abs}}=61.0 \pm 2.8$ mas $\left(d=16.39_{-0.72}^{+0.79} \mathrm{pc}\right)$ and a relative proper motion of $\mu_{\alpha}=-243 \mathrm{mas} \mathrm{yr}^{-1}$ and

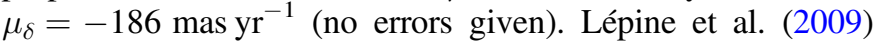
also obtained an optical spectrum to determine the unresolved spectral type as M7.0e with strong $\mathrm{H} \alpha$ emission.

At about the same time, we discovered a bright radio counterpart to LSPM J1314+1320AB with the Karl G. Jansky Very Large Array (VLA) of the National Radio Astronomy 
Observatory (NRAO), with the first observations reported in McLean et al. (2012). Given its brightness, LSPM J1314 $+1320 \mathrm{AB}$ appeared to be a good candidate for VLBI radio observations. Indeed, McLean et al. (2011) reported a VLBI detection of LSPM J1314+1320AB in the $3.5 \mathrm{~cm}$ band, noting that just one of the two components of this binary appeared to show radio emission. With just one epoch of VLBI data, it was impossible to tell which of the two components had been detected. The same paper also reported the results of multifrequency VLA observations and optical monitoring, confirming a persistent radio counterpart ( $\gtrsim 1 \mathrm{mJy}$ in the $3.5 \mathrm{~cm}$ band) and revealing variability with a period of about four hours. Most recently, Williams et al. (2015) reported Chandra and Swift X-ray detections in a multi-wavelength observation campaign. The angular resolution of the associated VLA observations was not sufficient to resolve the binary, but new radio variability patterns emerged from these observations, with slow variability overlaid by short flares lasting only a few minutes. In a near-infrared spectroscopic study of LSPM J1314 $+1320 \mathrm{AB}$, Schlieder et al. (2014) concluded that the system has an age of $\sim 30-200 \mathrm{Myr}$, and their preliminary mass estimates indicated that both components are near the substellar boundary.

Given the consistently detectable radio emission and one successful VLBI detection, we selected LSPM J1314+1320AB as a target for VLBI astrometric monitoring with the goal of measuring parallax and proper motion and then look for reflex motions caused by extrasolar planets. In a highly synergistic combination of data sets, we have analyzed our VLBI astrometric data together with Keck near-infrared adaptiveoptics monitoring, combining the advantages of absolute radio astrometry (even if on just one component) with relative infrared astrometry of both components (Dupuy et al. 2016, hereafter Paper II). The layout of this paper is as follows. In Section 2, we present the VLBI observations and data analysis. The contemporaneous Keck observations, the full astrometric fit, and all its parameters are presented in Paper II. In Section 3, we discuss the results of the VLBI astrometry, the radio properties of the two binary components, and prospects for comparison with Gaia data. In Section 4, we search for residual reflex motions of extrasolar planets, after taking into account parallax, proper motion, and binary orbital motion. Finally, in Section 5, we summarize our conclusions.

\section{OBSERVATIONS}

Observations were obtained with NRAO's Very Long Baseline Array (VLBA) under project BF108. The target was observed over a total of eight epochs, each with a duration of six hours. The observing $\log$ is listed in Table 1. At the beginning and end of each epoch, "geodetic-block" observations toward a selection of sources in the International Celestial Reference Frame catalog were carried out to improve the astrometric accuracy (Reid \& Brunthaler 2004). These were done in dual-band mode with eight S-band channels and eight $\mathrm{X}$-band channels.

In the main science block, rapid-switching phase-referencing observations were carried out using the nearby phase calibrator $\mathrm{J} 1309+1154$ at an angular separation of 1.8 from the target, with $40 \mathrm{~s}$ on target and $40 \mathrm{~s}$ on $\mathrm{J} 1309+1154$. The total onsource time for the target was $2.3 \mathrm{hr}$ per epoch. The observations were recorded with a bit rate of $2.048 \mathrm{Gbps}$, using the Roach Digital Backend and the polyphase filterbank
Table 1

VLBA Observing Log

\begin{tabular}{llcl}
\hline \hline Epoch & Date (UTC) & Day & Antennas $^{\mathrm{a}}$ \\
\hline BM327 & 2010 Apr 02 02:00-10:00 & -1324 & VLBA10+GBT \\
BF108A & 2013 Nov 16 13:30-19:30 & 0 & VLBA9(-FD) \\
BF108B & 2013 Nov 27 12:46-18:46 & 11 & VLBA9(-FD) \\
BF108C & 2013 Dec 31 10:00-16:00 & 45 & VLBA10 \\
BF108D & 2014 Feb 23 07:00-13:00 & 99 & VLBA10 \\
BF108E & 2014 Apr 29 02:40-08:36 & 164 & VLBA9(-NL) \\
BF108F & 2014 Jul 29 20:43-02:44 & 255 & VLBA10 \\
BF108G & 2014 Nov 04 14:15-20:16 & 353 & VLBA9(-MK) \\
BF108H & 2014 Nov 26 12:51-18:52 & 375 & VLBA9(-BR) \\
\hline
\end{tabular}

Note.

${ }^{\text {a }}$ VLBA10 is the full array, VLBA9 is missing one antenna as listed, the BM327 observation also involved the Green Bank Telescope (GBT).

digital signal-processing algorithm. The X-band setup for the science observations consisted of 16 channels with a bandwidth of $32 \mathrm{MHz}$ each, covering the frequency range of 7.052-7.324 GHz in dual polarization. The initial correlator position was estimated from earlier observations and then refined for later epochs.

When planning the sequence of observations, it was challenging to obtain an optimal temporal spacing of the eight observing epochs. Contrary to astrometric observations designed to obtain an optimal parallax measurement, our observations also had to consider the detection of astrometric reflex motions on an unknown timescale. In order to cover a wide range of epoch spacings (to probe a range of possible orbital periods), we scheduled the observations in multiples of 11 days near day $0,11,44,99,165,242,352$, and 374 , starting on 2013 November 16. However, external scheduling constraints meant that this sequence was not exactly realized (see Table 1).

The raw interferometric visibility data were calibrated and analyzed with the NRAO Astronomical Image Processing System (AIPS) software package using standard procedures. Initially, ionospheric delays were removed using measurements of the total electron content provided by Global Positioning System measurements, and all data were corrected using updated USNO Earth orientation parameters. Residual delays from clock drifts and zenith atmospheric delays were measured with the geodetic blocks and removed from the phase-reference data. Using observations of the strong calibration source J1800 +782 , we removed electronic delays and differences among the IF bands. Given the availability of a bright phase calibrator, we included a final determination of multi-band delays on this source and applied these to the entire data set. Then, we interpolated the phases for all rapid-switching reference sources.

After calibration, the visibility data were imaged with the AIPS task IMAGR, with the Briggs weighting parameter "robust" set to zero. The resulting average synthesized beam given the $(u, v)$ coverage of our observations was non-circular with a size of $2.9 \times 1.2$ mas, with the larger extension in decl. At the target distance, this corresponds to a linear resolution of about $10 \times 4 R_{\odot}$. Since $\mathrm{M}$ dwarfs have a radius of $\approx 0.1 R_{\odot}$, it is unlikely that these observations resolve any coronal structures in the target(s). However, the centroid of the radio position could still be affected somewhat in the case of unevenly distributed active regions, as discussed below. 
Table 2

2M1314 Detections

\begin{tabular}{|c|c|c|c|c|}
\hline Epoch & $\begin{array}{c}\text { R.A. (J2000) } \\
\text { 13:14:XX (J2000) }\end{array}$ & $\begin{array}{c}\text { decl. (J2000) } \\
+13: 19: X X(J 2000)\end{array}$ & $\begin{array}{l}\text { Beam Size } \\
\quad(\mathrm{mas})\end{array}$ & $\begin{array}{l}\text { Peak Flux Density } \\
\qquad\left(\mu \mathrm{Jy} \mathrm{bm}^{-1}\right)\end{array}$ \\
\hline BM327 & $20.1914692 \pm 0.0000013$ & $58.970242 \pm 0.000040$ & $2.9 \times 1.1$ & $525 \pm 17$ \\
\hline BF108A & $20.1238242 \pm 0.0000009$ & $58.277745 \pm 0.000027$ & $2.5 \times 1.0$ & $748 \pm 24$ \\
\hline BF108C & $20.1237042 \pm 0.0000010$ & $58.267385 \pm 0.000036$ & $3.3 \times 1.3$ & $592 \pm 23$ \\
\hline BF108D & $20.1208275 \pm 0.0000008$ & $58.270925 \pm 0.000026$ & $2.8 \times 1.1$ & $747 \pm 21$ \\
\hline BF108E & $20.1147535 \pm 0.0000009$ & $58.271672 \pm 0.000026$ & $2.7 \times 1.1$ & $728 \pm 22$ \\
\hline BF108H & $20.1107254 \pm 0.0000009$ & $58.123189 \pm 0.000036$ & $3.5 \times 1.2$ & $659 \pm 23$ \\
\hline
\end{tabular}

Note.

${ }^{\mathrm{a}}$ FWHM synthesized beam size in mas. The position angle was always within $5^{\circ}$ of the north-south direction, with the exception of epoch BF108G at $12^{\circ}$.

The asymmetric beam means that the absolute positions are better constrained in R.A. than in decl. However, this is not only governed by the synthesized beam shape. Residual tropospheric and ionospheric delays generally affect decl. more than R.A., and astrometric noise is expected to be higher in decl. than what would be expected from the beam shape alone (Reid \& Honma 2014). Combined with the fact that the parallax signature is larger in R.A. than in decl., the R.A. data dominate the parallax estimate.

To further improve our astrometric fit by increasing the time baseline, we also show our VLBA observations first reported by McLean et al. (2011). These were carried out in April 2010 (project ID BM327) with both the VLBA and the Green Bank Telescope to increase sensitivity. Since the associated geodeticblock observations were not used when we previously reported the VLBA detection, we re-reduced the BM327 data as described above.

\section{THE RADIO PROPERTIES AND MOTION OF LSPM J1314+1320AB}

\subsection{Detections and Flux Densities}

A single unresolved radio source was detected toward the target at all epochs, with peak flux densities ranging from 477 to $849 \mu \mathrm{Jy}$, with an average of $666 \mu \mathrm{Jy}$. The detailed fit results, including absolute positions, nominal position errors, and synthesized beam sizes are listed in Table 2. These position errors merely reflect the precision of the fit; they do not reflect systematic errors, which we will discuss below. While it was not immediately obvious that the detections always corresponded to the same component of the binary system, it became clear from the global astrometric fit (Paper II) that radio emission is indeed only detected from LSPM J1314+1320B.

The radio light curves suggest variability on timescales of weeks to months, but we refrain from discussing variability in more detail here. Given the known short-timescale variability in this source and the fact that we need to perform rapidswitching between the target and calibrator, our coverage of the short timescales is not ideal (see Williams et al. 2015). Interestingly, the VLBA flux densities are consistently lower by $\gtrsim 20 \%$ than those found in the VLA observations. Since we do not expect to resolve the stellar corona, there are two plausible explanations for this difference. The most likely explanation lies in uncertainties in the VLA/VLBA absolute flux scales, although noise in the phase-referencing data might scatter flux away from the target. Another effect that might contribute to this discrepancy is that the rapid phasereferencing VLBI observations do not provide good coverage on short timescales of minutes even though flaring variability (by factors of 2 ) has been observed toward this source (Williams et al. 2015). A dedicated study for radio variability at high time resolution on TVLM 513-46546 revealed even stronger, short flares (Wolszczan \& Route 2014). Given a low number of flares expected per epoch, we thus could easily have missed some short flares while observing the phase calibrator, and they may be bright enough to increase the time-averaged flux density when observed with the VLA. A detailed assessment would require simultaneous observations with the VLA and VLBA.

\subsection{Parallax and Proper Motion}

In contrast to our earlier work on TVLM 513-46546 (Forbrich et al. 2013), the astrometric fit for LSPM J1314 $+1320 \mathrm{AB}$ has to take into account not only the parallax and proper motion, but also the orbital motion in the binary system. With just one of the two components detected in the radio, the combination radio data with resolved near-infrared monitoring (Paper II) provide mutual constraints on the positions for both components. The VLBA data, with much higher (and absolute) astrometric accuracy, determine the parallax and proper motion, while the Keck data, with the detection of both stars, determine the binary orbit and other properties of the system. In particular, the Keck data constrain the shape of the orbit while the VLBA data constrain the semimajor axis of the orbit for the secondary (a parameter unconstrained by the Keck data alone). The full astrometric fit, including the determination of the orbit, is discussed in Paper II.

In Figure 1, we show the R.A. and decl. residuals as a function of time, subtracting the orbital and proper motion (to highlight the parallax) and additionally subtracting the parallax (to show residuals that constrain planetary perturbations). The parallax of LSPM J1314+1320AB is $\pi_{\mathrm{abs}}=57.975 \pm 0.045$ mas, i.e., with an error of $\leqslant 0.08 \%$, corresponding to a distance of $d=17.249 \pm 0.013 \mathrm{pc}$. The error of $45 \mu \mathrm{as}$ is 60 times better than for the previous parallax determination $(\pi=61.0 \pm 2.8$ mas, Lépine et al. 2009, which was estimated from a relative measurement). Similarly, our constraints on the absolute proper motion improve on the earlier determination. We find $\mu_{\alpha \cos \delta}=-247.99 \pm 0.10$ mas and $\mu_{\delta}=-183.58 \pm 0.22$ mas. Given the difficulties of obtaining 

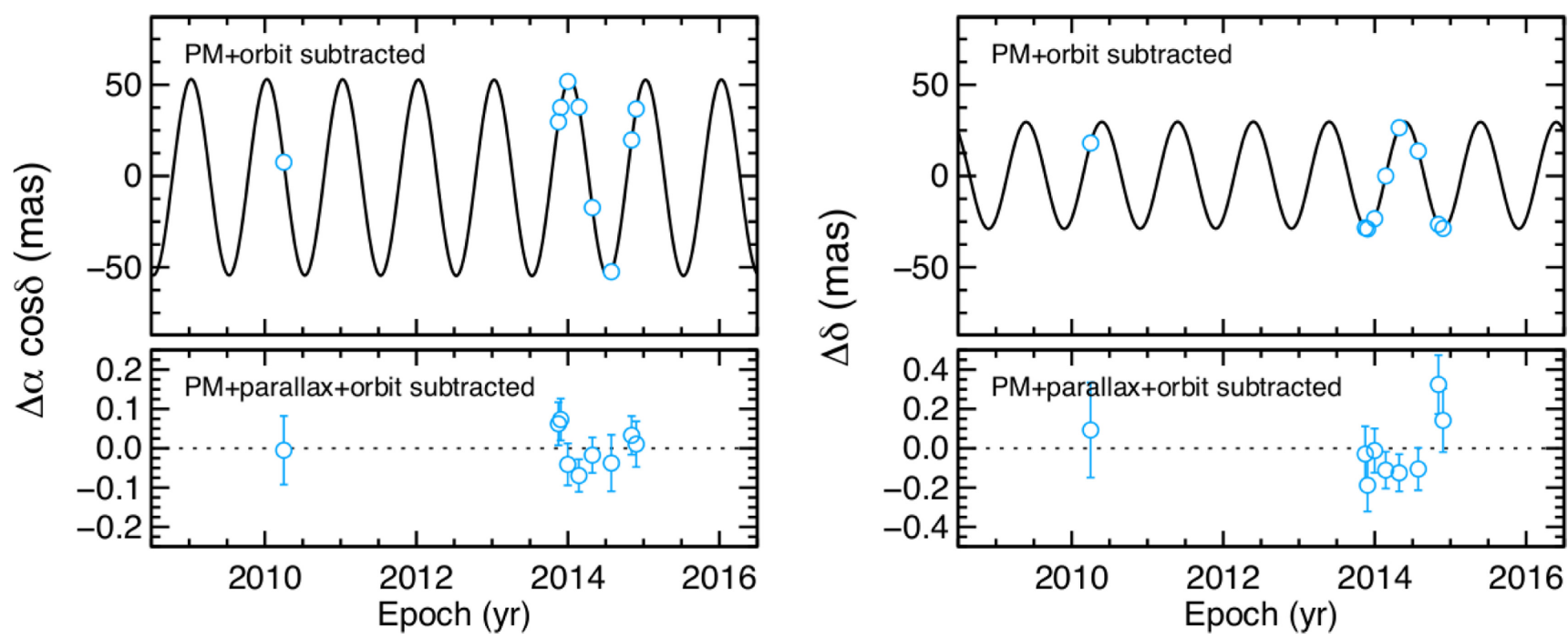

Figure 1. Astrometric residuals in R.A. ( $\alpha \cos \delta$, left panel) and decl. ( $\delta$, right panel). The upper plots show the effect of the parallax, when proper motion and orbital motion are subtracted. The lower plots show the astrometric residuals when additionally subtracting the parallax. The error bars indicate $1 \sigma$ errors.

an absolute proper motion measurement from optical (relative) measurements, the match to the previous results is very good.

\subsection{Prospects for Cross-checking with Gaia}

LSPM J1314+1320AB will be an important test case for the Gaia mission, as it will be possible to directly compare their astrometric calibration against the absolute astrometry presented here. The uncertainty of our absolute parallax measurement is $45 \mu$ as. For a single star with half of the optical brightness of the combined system LSPM J1314+1320AB $\left(V-I_{C}=3.89 \mathrm{mag}\right.$ and $V=15.83 \mathrm{mag}$, Lépine et al. 2009), Gaia is expected to deliver a nominal end-of-mission parallax uncertainty that is better by a factor of about two (European Space Agency 2016), at $\sigma_{\pi}=20 \mu$ as. The main complicating factor that may increase this uncertainty will be that LSPM $\mathrm{J} 1314+1320 \mathrm{AB}$ is, of course, a binary with a separation of $<0$ ". 2 , with about half of the 9.6-year orbit monitored during the Gaia mission. The expectation is, however, that Gaia will "systematically resolve double stars down to separations of $\sim 0$ " $1 . "$

In a similar test for the previous astrometric mission Hipparcos, VLBI results confirmed a $10 \%$ error in the combined parallax of the Pleiades cluster stars (Melis et al. 2014). Distance measurements derived with several other techniques had also disagreed with the Hipparcos result. The discrepancy had been large enough to imply severe problems for astrophysical models of young star stellar evolution. Thus, direct VLBI measurement of the absolute parallax can be of great value in the Gaia era.

For brown dwarfs, which are fainter and cooler than LSPM $\mathrm{J} 1314+1320 \mathrm{AB}$, the astrometric performance of the VLBA will be superior to that of Gaia, whenever the target shows sufficiently bright radio emission. In the absence of a correlation between the optical brightness of a brown dwarf and its radio emission, this is best shown in a concrete example. For LSPM J0036+1821, an L3.5 dwarf with radio emission (Berger 2002), the Gaia astrometric performance model predicts an uncertainty of the parallax measurement of $\sigma_{\pi}=115 \mu$ as, more than twice the uncertainty that we have reached for LSPM J1314+1320AB.

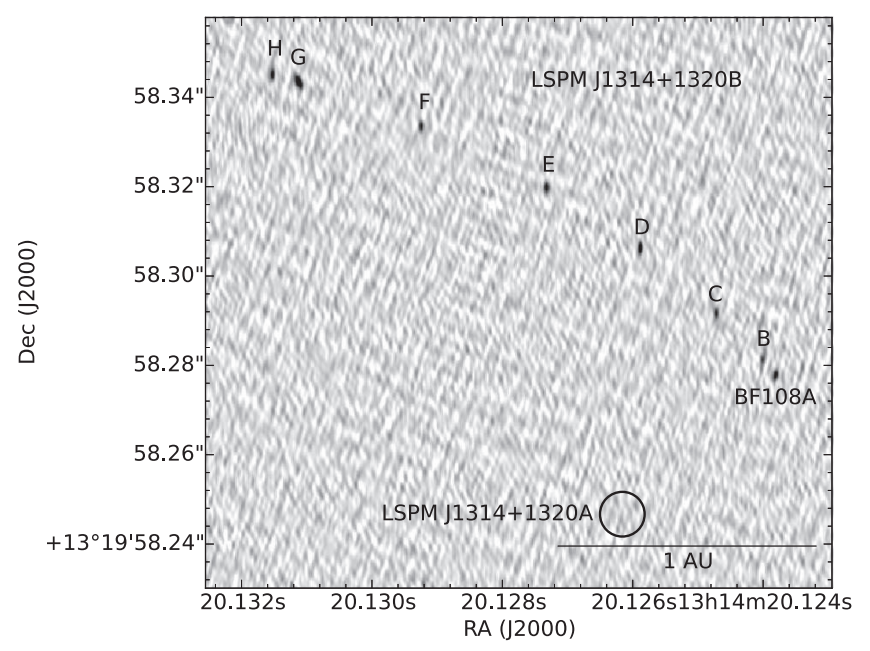

Figure 2. Co-added images of VLBA epochs BF108A-H, centered on the predicted position of the primary component LSPM J1314+1320A. The primary remains undetected; its position is marked with a circle of radius 5 mas. All eight detections of the secondary, starting with the westernmost point, are clearly visible, and the shape of the orbit is recognizable (see Figure 1 in Paper II).

\subsection{Upper Limits to the Radio Emission of LSPM J1314+1320A}

While the first VLBA observations of McLean et al. (2011) produced just one radio counterpart toward LSPM J1314 $+1320 \mathrm{AB}$, the non-detection of the other component could have been due to variability. Similarly, when we found a single radio source in all eight new epochs, it was not immediately clear whether one component was always radio-bright and the other radio-faint. This ambiguity was resolved by the full astrometric fit, taking into account parallax, proper motion, as well as orbital motion, and combining the VLBA and Keck data (Paper II). The fit is consistent with a continuous detection of the secondary component and a continuous non-detection of the primary component. The inclusion of the 2010 observations reported by McLean et al. (2011) allows us to additionally state that also then only the secondary component was detected with 
the VLBA. Whatever is causing this difference in the radio luminosity of the two components, it appears to be stable on timescales of years.

To derive an upper limit for the radio emission of LSPM $\mathrm{J} 1314+1320 \mathrm{~A}$, we have used the full astrometric fit to predict its position at the various times of the VLBA observations. The nominal positional error for these predicted positions is between 0.2 and 0.5 mas, i.e., generally smaller than the synthesized beam size. This allows us to make a more sensitive image by coadding the different radio epochs (Figure 2), all aligned at the respective position of LSPM J1314+1320A, with a positional error within the half-power synthesized beam width. Since the position uncertainty for the first epoch in 2010 is slightly larger than the synthesized beam, we excluded it from this approach. In the individual epochs, the local $1 \sigma$ noise at the predicted positions of component A was $21-26 \mu \mathrm{Jy}$, providing a $3 \sigma$ detection limit for the radio emission of component A of $\lesssim 63-78 \mu \mathrm{Jy}$ per epoch. With the co-added data from eight epochs, we obtain an upper limit for LSPM $\mathrm{J} 1314+1320 \mathrm{~A}$ 's radio emission of $\lesssim 22 \mu \mathrm{Jy}(3 \sigma)$, indicating that LSPM J1314+1320B is brighter than LSPM J1314 $+1320 \mathrm{~A}$ by a factor of $\gtrsim 30$ (compared to a factor of $\gtrsim 10$ when based on the individual epochs), assuming continuous emission.

Even though the stars have masses that are consistent to within $\approx 2 \%$ (Paper II), they appear to have vastly differing radio emission. One possibility to explain this discrepancy would be that in spite of otherwise almost identical properties, the rotational velocities of the two components and/or their prior evolution are significantly different, which could result in different magnetic field configurations. Rotation velocity is well known as a main energy reservoir for stellar activity. While differences in rotational velocities (when measured as $v \sin i$ ) have been found in several nearby very-low-mass binaries (Konopacky et al. 2012), no such resolved $v \sin i$ measurements have yet been published for LSPM J1314 +1320 AB. McLean et al. (2011) reported an unresolved measurement of $v \sin i=45 \pm 5 \mathrm{~km} \mathrm{~s}^{-1}$, indicative of rapid rotation in at least one component. Preliminary results from a resolved measurement indicate that the two stars have very similar $v \sin i$, close to the unresolved measurement (P. K. G. Williams et al. 2016, in preparation). However, it is not clear how large a difference in $v \sin i$ would be required to explain the factor of $\gtrsim 30$ in radio luminosity between two otherwise almost identical stars.

Differences in $v \sin i$ could come from different rotational velocities or different alignments of the rotational axes (or both). In the latter case, the differences in emission could also occur if the radio emission is highly beamed, as suggested by work on the electron-cyclotron maser emission, which is thought to be occurring on ultracool dwarfs (e.g., Hallinan et al. 2007; Nichols et al. 2012). The question of aligned rotational axes in binaries is an active area of research (e.g., Albrecht et al. 2014) with no clear answers. Also, radio surveys provide no empirical evidence on how strongly the radiation is beamed (e.g., McLean et al. 2012). In either case, very different rotational velocities or different axis alignments of two otherwise almost identical stars in a coeval binary, would have interesting implications for the formation and evolution of very-low-mass stars.

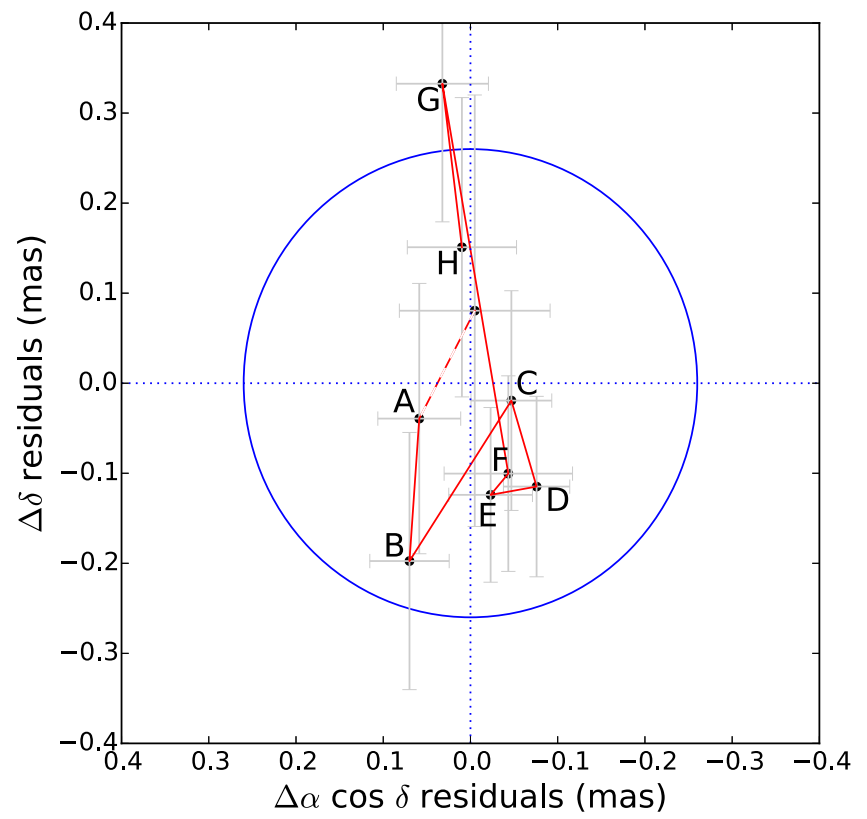

Figure 3. 2D astrometric residuals of LSPM J1314+1320AB, after parallax, proper motion, and orbital motion have been subtracted. The error bars indicate the nominal errors with the error floors added in quadrature. The blue circle indicates the $3 \sigma$ radial (2D) rms, used as an upper limit for the presence of reflex motions.

\section{UPPER LIMITS TO THE PRESENCE OF PLANETS IN ORBIT AROUND LSPM J1314+1320B}

\subsection{Assessment of Astrometric Residuals}

To search for potential signatures of extrasolar planets, we assume that any residual astrometric trends, after the parallax, proper motion, and the binary orbit have been taken into account, may be due to the reflex motions from one or more planets. In the absence of a clear trend, the scatter of the residuals of the astrometric fit provides significant limits to the presence of planets.

In our data, there is no obvious systematic trend in the residuals. Each step of the Markov chain has an independent realization of the residuals about that particular astrometric solution. For each epoch, we thus calculated the mean and rms of that data point's residual with respect to all MCMC models. The residuals in R.A. and decl. show no obvious trend as a function of time in Figure 1, nor when considering the 2D residuals shown in Figure 3, where systematic trends due to reflex motion could also show up. The $3 \sigma$ rms noise in the 2D residuals is 0.26 mas, and in R.A. $\cos \delta$ and decl. it is 0.15 mas and 0.50 mas, respectively. Overall, these residuals are not unusually high when compared to our previous observations (e.g., Forbrich et al. 2013).

Depending on the eccentricity of a hypothetical planetary orbit, its orientation relative to the line of sight, and the spacing of observations within the orbital period, the observed reflex motion could lie below these limits. In the following, we primarily use the 2D residuals to define the limit. In Section 4.4, we will additionally use simulated planetary orbits and associated reflex motions to assess the detection completeness.

\subsection{Discussion of Errors}

Realistic VLBA position errors usually exceed the formal position fitting uncertainties. To account for this, we introduce 
"error floors" in both R.A. and decl. to represent systematic errors. These are treated as parameters in the full parallax/ proper motion/orbit fit (Paper II), and we find $\sigma_{\alpha \cos \delta}=0.064_{-0.024}^{+0.037}$ mas and $\sigma_{\delta}=0.180_{-0.067}^{+0.106}$ mas. These error floors should be added in quadrature to those given in Table 2.

If the reflex motion due to a planet does not average out over the course of the experiment, then the reflex motion signal may contribute to the error floors, and as a result the reflex motion will appear less significant than it actually is. However, the temporal shape of the reflex motion signal will remain unaffected. Upper limits on reflex motions are thus doubly conservative: on the one hand, the residuals contain unmodeled effects other than planets, and on the other hand the significance of any reflex motion signal may be underestimated.

In the following analysis, we assume that the centroid of the radio emission coincides with the centroid of the stellar photosphere. This would require that the active regions that are producing the radio emission either fill enough of the stellar photosphere that the centroid remains stationary even though the star is rotating or that the active regions are stationary close to the poles of the star. If there are just a few active regions, the radio centroid will wobble around the optical/infrared centroid.

Since our full model of the two binary components has produced estimates of their radii, from a comparison of their masses and their bolometric luminosities with evolutionary models, we can estimate the maximum amplitude of this wobble by assuming that the active regions are at the photospheric radius. The observability of this effect and its timescale depend on the currently unknown stellar rotation period and the inclination of the rotation axis. The estimated radius of $r \approx 1.8 R_{\text {jup }}$ (Paper II) corresponds to an angular size of $54 \mu \mathrm{as}$ at the distance of LSPM J1314+1320AB. Thus, asymmetric coronal structures could cause a wobble of $<54 \mu$ as, which is on the order of the R.A. error floor and thus conservatively accounted for in our analysis.

\subsection{Inferred Astrometric Limits on the Presence of Planets}

The upper limits to the reflex motion translate into an excluded region in the phase space of planetary mass and orbital period via Kepler's Third Law. For a discussion of the limits for circular planetary orbits, see Eisner \& Kulkarni (2001b) and Forbrich et al. (2013). Basically, the reflex motion $\theta$, as a function of orbital radius $a$, is determined by the observationally constrained orbital period $P$ as follows.

$$
\theta=\frac{a}{d} \frac{m_{c}}{m_{*}}=\frac{m_{c}}{d}\left(\frac{G P^{2}}{4 \pi^{2}\left(m_{*}+m_{c}\right)^{2}}\right)^{1 / 3},
$$

where $d$ is the distance, $m_{c}$ is the companion mass, and $m^{*}$ is the mass of the central object. The $2 \mathrm{D}$ residuals thus result in an excluded region shown as a shaded area in Figure 4; we note that the lower R.A. residuals exclude a subset of orbits with even lower planetary mass limits, while the limit on reflex motions only in decl. is higher. The $3 \sigma$ upper limit from the 2D astrometric residuals excludes planets of masses $0.7-10 M_{\text {jup }}$ for orbital periods of 600 days to 10 days, respectively. The limiting planetary mass as a function of orbital period can be described as $M \approx 47 \times P(d)^{-2 / 3} M_{\text {jup}}$, where $P(d)$ is the orbital period in days. While Figure 4 does not show the long
Orbital radius $(A U)$

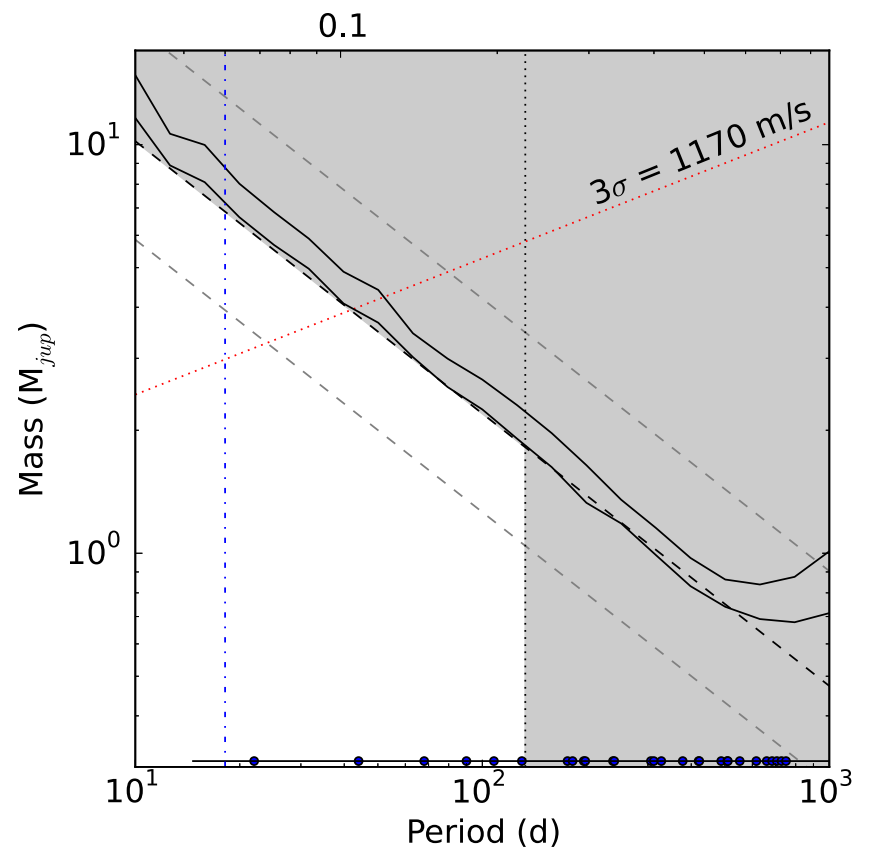

Figure 4. Limits to the presence of a hypothetical single extrasolar planet that would cause reflex motions of LSPM J1314+1320B above the observed astrometric residuals (see the text). The gray areas denote the parameter space that can be ruled out. The black dashed line indicates the limit corresponding to the $3 \sigma 2 \mathrm{D} \mathrm{rms}$ of the residuals, while the gray dashed lines indicate the $3 \sigma \mathrm{rms}$ limits separately in decl. (upper line) and R.A. (lower line). Depending on the orbit geometry and in particular the eccentricity, the limit is between these two extremes. The two black continuous lines show $95 \%$ detection completeness of simulated orbits that are circular (lower curve) or have $e=0.6$ (upper curve). As an additional constraint, we point out that orbits with $P>133$ days are unstable in this system (based on the orbit solution from Paper II, see the text) and thus ruled out, too. The area above the red dotted line is the approximate phase space, with $M \sin i$ instead of $M$, that could additionally be ruled out in radial velocity monitoring when assuming an uncertainty for rapidly rotating stars $\left(3 \sigma=1170 \mathrm{~m} \mathrm{~s}^{-1}\right)$ based on the work by Blake et al. (2010). The blue dots at the bottom of the plot are shown as a measure of our coverage of parameter space. Dots are placed at orbital periods $P$ where there is a time baseline of $P / 2$ among the VLBA observing epochs, with lines indicating intervals $[1 / 4 P, 3 / 4 P]$, i.e., where there is good sensitivity for the maximum reflex motion caused by such orbits. Finally, the vertical blue dashed-dotted line indicates the outer radius of the habitable zone around the star according to Selsis et al. (2007).

periods traced by the inclusion of the 2010 data, no significant deviations from the model are seen on those timescales either (Figures 1 and 3). Given our observational scheduling, we are sensitive to a nearly continuous range of orbital periods from 10 to 1000 days, since for any period in this range we have at least one pair of measurements that avoid near integer multiples of orbits (shown in Figure 4). A quantitative assessment of our sensitivity to orbital periods requires simulations of planetary orbits and their reflex motions, which we do in Section 4.4.

At small orbital radii, our experiment is probing the outer habitable zone of LSPM J1314+1320B, which extends to a distance of $\sim 0.06$ au from the central object (Selsis et al. 2007; shown in Figure 4). Our VLBA astrometry allows us to exclude planets of $\approx 7 M_{\text {jup }}$ in the outer habitable zone.

\subsection{Assessment of Planet-detection Completeness}

To assess the detection completeness to different planetary orbits in detail, we have simulated orbits over the full range of 
the astrometric model of the binary, making use of the mass and distance of LSPM J1314+1320B. We have simulated $10^{6}$ planetary orbits at random viewing angles and with eccentricities of $e=0-0.6$, covering our parameter space in orbital periods and planetary mass (10-1000 days and 0.1-10 $\left.M_{\text {jup }}\right)$. In the simulated astrometric data, we approximately account for proper motion by first subtracting linear fits of the systematic motion in both R.A. and decl. to then assess the residuals, again separately in R.A. and decl. We adopt a simple detection criterion where the residuals in either R.A. or decl. have to be larger than the $3 \sigma$ limits reported above. Finally, we calculated the $95 \%$ detection fraction from the binned simulation results.

The results of these simulated planetary orbits are overplotted in Figure 4 for circular orbits as well as elliptical orbits with $e=0.6$. The estimated $95 \%$ detection completeness corresponds very well with the criterion derived from the $2 \mathrm{D}$ $3 \sigma$ astrometric residuals above, across almost the entire relevant range of orbital periods, as expected from our observation intervals. Only at the shortest and longest orbital periods do the limits rise toward higher masses. Shorter periods that we cannot resolve with our observing sequence would show up as extra astrometric noise throughout the experiment and would be absorbed in our error floors. Orbital periods much longer than our spanned observations would add a constant position offset, and more generally would add to the apparent astrometric noise. Additionally, our setup is slightly less complete for elliptical orbits when compared to circular orbits, i.e., their limits generally lie at higher masses for a given orbital period.

Note that the simple detection criterion in this test does not account for the degeneracy of orbital periods of one year with the Earth's orbit, which defines the parallax measurement. However, it is very unlikely that a planet's reflex motion, measured at multiple intervals, would match up with the parallax motion in both R.A. and decl. Additional tests with simulated orbits suggest that this effect only slightly reduces our sensitivity to such planets, but we refrain from discussing this in more detail since we can rule out such orbits from orbit stability considerations within this binary system.

\subsection{Stability of Planetary Orbits}

The limit on long orbital periods can be constrained with information from the full astrometric fit (Paper II), by including stability considerations of a planet orbiting one component of a binary system. Using the empirical expression for the critical semimajor axis $\left(a_{c}\right)$ of such orbits from Holman \& Wiegert (1999), above which planetary orbits would not be stable, we obtain $a_{c}=0.23 \mathrm{au}$, corresponding to an upper limit of stable planetary orbital periods of 133 days. While our planetary mass limit based on the absence of reflex motions is already very low for these long orbital periods (for example, a planet of $1 M_{\text {jup }}$ and an orbital period of 330 days can be excluded), this constraint rules out a substantial additional region in the phase space of planetary mass versus orbital period, as shown in Figure 4. Only the white area in that figure remains as potential phase space for hypothetical planets in orbit around LSPM $\mathrm{J} 1314+1320 \mathrm{~B}$.

\subsection{Comparison with other Planet-detection Methods}

Our observations provide constraints on the presence of extrasolar planets in a parameter range that is otherwise impossible to access. The principal comparison here is with RV surveys for planets, which are sensitive to $M \sin i$. The sensitivity of this method is biased toward close-in planets (e.g., Eisner \& Kulkarni 2001a). While no RV monitoring of LSPM J1314+1320AB has yet been published, we now assess what fraction of the parameter space shown in Figure 4 may be accessible with such measurements. The main challenge is the high $v \sin i=45 \mathrm{~km} \mathrm{~s}^{-1}$ (McLean et al. 2012).

In an RV survey of ultracool dwarfs, Blake et al. (2010) reach an RV precision of $100-300 \mathrm{~m} \mathrm{~s}^{-1}$ for slowly rotating stars with $v \sin i<30 \mathrm{~km} \mathrm{~s}^{-1}$. For the 13 ultracool dwarfs in their sample with $v \sin i>30 \mathrm{~km} \mathrm{~s}^{-1}$ (all L dwarfs), the median uncertainty is $390 \mathrm{~m} \mathrm{~s}^{-1}$ with a standard deviation of $285 \mathrm{~m} \mathrm{~s}^{-1}$. Using this uncertainty to assess the feasibility of RV planet searches toward LSPM J1314+1320AB, the corresponding $3 \sigma$ limit to the detection of planets at $1170 \mathrm{~m} \mathrm{~s}^{-1}$ is indicated in Figure 4. A comparison with our constraints from astrometric monitoring reveals that RV monitoring could produce slightly better constraints on lower-mass planets at small orbital radii of $\lesssim 0.08 \mathrm{au}$, and, hence, the two methods are complementary.

Absolute astrometry is not necessary to conduct reflex motion searches for extrasolar planets. With relative astrometry in $I$ band, such a search has been carried out toward 20 ultracool dwarfs by Sahlmann et al. (2014), reaching a median $3 \sigma$ astrometric residual of 0.60 mas with a standard deviation of 0.16 mas. The $3 \sigma 2 \mathrm{D}$ residual of 0.26 mas that we find toward LSPM J1314+1320B thus is significantly better, but at the same time a larger sample of targets is more easily available in the near-infrared than in the radio range.

\section{SUMMARY AND CONCLUSIONS}

We presented multi-epoch VLBA observations of the ultracool dwarf binary LSPM J1314+1320AB. In combination with Keck imaging data, a complete orbit solution was obtained, which is discussed in Paper II. In this paper, we have used the VLBA data and the orbit solution to determine the radio properties of both binary components, a parallax, proper motions, and astrometric limits to the presence of extrasolar planets. Our conclusions can be summarized as follows.

1. The radio emission observed on milliarcsecond scales toward LSPM J1314+1320AB is entirely due to its secondary component (B), which is detected at an average flux density of $666 \mu \mathrm{Jy}$. An upper limit of $\lesssim 22 \mu \mathrm{Jy} \mathrm{bm}^{-1}$ $(3 \sigma)$ is derived for continuous radio emission of the primary (A). Given that our full astrometric fit yields masses for the two components that are consistent to within $\approx 2 \%$ (Paper II), this difference by a factor of $\gtrsim 30$ is remarkable. This could point to differences in stellar rotation as an underlying factor governing stellar activity, or to differences in the evolution of stellar rotation over time, both potentially resulting in different magnetic field configurations. Alternatively, the radio emission could be beamed toward us for the secondary but not for the primary if the rotation axes of the two stars are not aligned. Resolved $v \sin i$ measurements of the two stars could potentially elucidate these issues (P. K. G. Williams et al. 2016, in preparation).

2. The VLBA data allow us to measure the absolute parallax toward LSPM J1314+1320AB as 
$\pi_{\text {abs }}=57.975 \pm 0.045$ mas, with an uncertainty 60 times lower than previous measurements and corresponding to a distance of $d=17.249 \pm 0.013$ pc. Also, we obtained an absolute proper motion, which is similar, but more accurate than, the previous estimate. Our absolute astrometry will constitute an important cross-check for the astrometric calibration of Gaia.

3. Given the full orbit solution and characterization of the binary components in Paper II, we can assess the impact of marginally resolved coronal structures on the centroid of the radio emission. The estimated radius of the secondary, and thus the maximum amplitude of any astrometric wobble due to asymmetric coronal structures, corresponds to an angular size of $54 \mu$ as at the distance of LSPM J1314+1320AB. This is on the order of the lowest derived (R.A.) error floor and thus is conservatively accounted for in our analysis.

4. We find no obvious signature of reflex motions due to the presence of one or more extrasolar planets in orbit around LSPM J1314+1320B. A $3 \sigma$ limiting planetary mass as a function of orbital period is given as $M\left(M_{\text {jup }}\right)=47 \times P(d)^{-2 / 3}$ (where $P(d)$ is the orbital period in days), ruling out, for example, a planet of $2.2 M_{\text {jup }}$ with an orbital period of 100 days. Applying our full orbit solution for the binary, we additionally obtain a general upper limit for orbital radii $(a \lesssim 0.23$ au) of stable planetary orbits in this binary system. Most of the parameter range of the derived constraints is inaccessible to RV surveys, but they do add additional limits on lower-mass planets at small orbital radii of $\lesssim 0.08$ au.

5. Finally, the combination of VLBA and Keck data has proven to be very powerful in this project. The VLBA data provide excellent absolute astrometry of, in this case, one binary component, and the Keck data provide excellent relative astrometry of both components. In combination, the two data sets provide the basis for a full astrometric fit to an ultracool-dwarf binary at unprecedented accuracy.
The National Radio Astronomy Observatory is a facility of the National Science Foundation operated under cooperative agreement by Associated Universities, Inc. J.F. acknowledges helpful discussions with Andreas Brunthaler, Peter Williams, Alex Wolszczan, Andreas Seifahrt, and Elisabeth Newton.

Facilities: VLBA, Keck.

\section{REFERENCES}

Albrecht, S., Winn, J. N., Torres, G., et al. 2014, ApJ, 785, 83

Baraffe, I., Homeier, D., Allard, F., \& Chabrier, G. 2015, A\&A, 577, A42 Berger, E. 2002, ApJ, 572, 503

Berger, E., Ball, S., Becker, K. M., et al. 2001, Natur, 410, 338

Blake, C. H., Charbonneau, D., \& White, R. J. 2010, ApJ, 723, 684

Bower, G. C., Bolatto, A., Ford, E. B., \& Kalas, P. 2009, ApJ, 701, 1922

Dressing, C. D., \& Charbonneau, D. 2015, ApJ, 807, 45

Dupuy, T. J., Forbrich, J., Rizzuto, A., et al. 2016, ApJ, 827, 23

Dupuy, T. J., Liu, M. C., Leggett, S. K., et al. 2015, ApJ, 805, 56

Eisner, J. A., \& Kulkarni, S. R. 2001a, ApJ, 561, 1107

Eisner, J. A., \& Kulkarni, S. R. 2001b, ApJ, 550, 871

European Space Agency 2016, Gaia: Science Performance, http://www. cosmos.esa.int/web/gaia/science-performance

Forbrich, J., \& Berger, E. 2009, ApJ, 706, L205

Forbrich, J., Berger, E., \& Reid, M. J. 2013, ApJ, 777, 70

Hallinan, G., Bourke, S., Lane, C., et al. 2007, ApJ, 663, L25

Holman, M. J., \& Wiegert, P. A. 1999, AJ, 117, 621

Kirkpatrick, J. D., Henry, T. J., \& Irwin, M. J. 1997, AJ, 113, 1421

Konopacky, Q. M., Ghez, A. M., Fabrycky, D. C., et al. 2012, ApJ, 750, 79

Law, N. M., Hodgkin, S. T., \& Mackay, C. D. 2006, MNRAS, 368, 1917

Lépine, S., \& Shara, M. M. 2005, AJ, 129, 1483

Lépine, S., Thorstensen, J. R., Shara, M. M., \& Rich, R. M. 2009, AJ, 137,4109

McLean, M., Berger, E., Irwin, J., Forbrich, J., \& Reiners, A. 2011, ApJ, 741, 27

McLean, M., Berger, E., \& Reiners, A. 2012, ApJ, 746, 23

Melis, C., Reid, M. J., Mioduszewski, A. J., Stauffer, J. R., \& Bower, G. C. 2014, Sci, 345, 1029

Nichols, J. D., Burleigh, M. R., Casewell, S. L., et al. 2012, ApJ, 760, 59

Reid, M. J., \& Brunthaler, A. 2004, ApJ, 616, 872

Reid, M. J., \& Honma, M. 2014, ARA\&A, 52, 339

Sahlmann, J., Lazorenko, P. F., Bouy, H., et al. 2016, MNRAS, 455, 357

Sahlmann, J., Lazorenko, P. F., Ségransan, D., et al. 2014, A\&A, 565, A20

Schlieder, J. E., Bonnefoy, M., Herbst, T. M., et al. 2014, ApJ, 783, 27

Selsis, F., Kasting, J. F., Levrard, B., et al. 2007, A\&A, 476, 1373

Williams, P. K. G., Berger, E., Irwin, J., Berta-Thompson, Z. K., \& Charbonneau, D. 2015, ApJ, 799, 192

Wolszczan, A., \& Route, M. 2014, ApJ, 788, 23 